\title{
Concurrent chemoradiotherapy was associated with a higher severe late toxicity rate in nasopharyngeal carcinoma patients compared with radiotherapy alone: a meta-analysis based on randomized controlled trials
}

\author{
Cheng-run Du, Hong-mei Ying ${ }^{*}$, Fang-fang Kong, Rui-ping Zhai and Chao-su Hu
}

\begin{abstract}
Background: To investigate the incidence and risk of severe late toxicity with concurrent chemoradiotherapy (CCRT) in nasopharyngeal carcinoma patients.

Methods: Eligible studies included prospective randomized controlled trials (RCTs) evaluating CCRT versus radiotherapy alone in patients with nasopharyngeal carcinoma and in which data on severe late toxicities were available. Random effects or fixed effect models were applied to obtain the summary incidence, relative risks (RRs) and $95 \%$ confidence intervals (Cls).

Results: Five RCTs with 1102 patients with NPC were included in this analysis. The summary incidence of overall severe late toxicities in patients receiving CCRT was 30.7\% (95\% Cl, 18-47.2\%) and the incidence of radiotherapy alone group was $21.7 \%$ (95\% Cl, 13.3-33.4\%). The use of concurrent chemotherapy was associated with an increased risk of severe late toxicities, with a RR of $1.349(95 \% \mathrm{Cl}, 1.108-1.643 ; \mathrm{P}=0.005)$. As for specific late toxicity, CCRT significantly increased the risk of ear deafness/otitis $(R R=1.567 ; 95 \% \mathrm{Cl}, 1.192-2.052)$, but other late toxicities were not significantly different. Patients receiving concurrent chemotherapy regimens with 3-week high-dose cisplatin (HC) have a higher risk of ear deafness/otitis ( $R R=1.672 ; 95 \% \mathrm{Cl}, 1.174-2.382 ; \mathrm{P}=0.026)$. However, there was no significant increase in the RR of severe ear complication with the addition of non-3-week high-dose cisplatin (nonHC) regimens $(\mathrm{RR}=1.433 ; 95 \% \mathrm{Cl}$, $0.946-2.171 ; P=0.095)$.
\end{abstract}

Conclusion: With the present evidence, the addition of concurrent chemotherapy seems to increase the risk of severe late toxicities in patients with NPC, especially when using $\mathrm{HC}$ regimen for the occurrence of severe ototoxicity.

Keywords: Nasopharyngeal carcinoma, Concurrent chemoradiotherapy, Severe late toxicities, Meta-analysis

\section{Background}

Nasopharyngeal carcinoma (NPC) is sensitive to radiation, but the outcomes of patients with advanced-stage NPC were unsatisfactory [1,2]. Given the chemosensitive nature of NPC, the combination of chemotherapy and radiation has been investigated for advanced NPC. No consensus on the optimal protocol had been reached until the Intergroup 0099 study [3], which demonstrated

\footnotetext{
* Correspondence: yinghongmei2011@sina.com

Department of Radiation Oncology, Fudan University Shanghai Cancer Center, 270 Dongan Road, 200032 Shanghai, People's Republic of China
}

that concurrent chemoradiotherapy (CCRT) plus adjuvant chemotherapy using cisplatin-based chemotherapy was superior to radiation therapy alone. Subsequently, evidence increasingly mounted from randomized trials [4-8] and meta-analyses [9] confirming the advantage of CCRT.

Although the superiority of CCRT for NPC has been indisputable, there are concerns about the side effects caused by the combination of concurrent chemotherapy and radiotherapy. Severe acute toxicities could impair the compliance of treatment. Severe late toxicities (grade 
3 or 4) can be life-threatening or significantly erode the patient's quality of life (QoL) and functional status [10]. As the overall survival for NPC patients improves, late toxicities may become more frequent. Concurrent chemotherapy was found to increase the acute toxicities during radiotherapy [6-8]. However, the overall incidence and risk of severe late toxicities with additional concurrent chemotherapy in NPC remain unknown because many of the published data were based on studies that were either retrospective, or with inadequate duration of observation for full assessment of long-term toxicity, or were obscured by treatment heterogeneity [11-13].

We conducted this meta-analysis of prospective randomized controlled trials (RCTs) reporting severe late toxicities to investigate the overall incidence and risk of severe late toxicities with CCRT in NPC to help clinicians target possible severe late effects that need special attention during patient follow-up.

\section{Methods}

We identified and selected relevant studies by searching the databases: Web of knowledge, Embase, Cochrane library databases and Pubmed updated to August 2014. The search was conducted by using the keywords "nasopharyngeal carcinoma", "radiotherapy", "chemotherapy", and "chemoradiotherapy" and was limited to human studies and clinical trials published in English. The reference list of each article obtained was checked for further potential studies. Only RCTs in which CCRT was compared with radiotherapy alone in NPC patients and the detailed data on severe late toxicities were reported, were eligible for inclusion in the meta-analysis. The report quality of the clinical trials was assessed and calculated using Jadad scale including randomization, double-blinding, and withdrawals as previously described [14].

Data from the studies were extracted independently by two investigators (CD and FK) using standardized data forms and disagreement was resolved by consensus. The following information was extracted: author's name, publication year, number of enrolled subjects, treatment arms, number of patients in each group, the duration of follow-up and events of severe late toxicities. This study was performed with the approval of Institutional Review Boards of Fudan University Shanghai Cancer Center.

\section{Statistical analysis}

The pooled analysis of incidence and RR were performed with Open Meta-Analyst software version 3.13. The publication bias was tested with Stata/SE 12.0. A classic half-integer continuity correction was applied to obtain the relative risk (RR) and variance for studies reporting zero events in a treatment or control arm. Betweenstudy heterogeneity was estimated using chi-squared and $\mathrm{I}^{2}$ tests. Heterogeneity was considered statistically significant when $\mathrm{P}<0.05$ or $\mathrm{I}^{2}>50 \%$. If heterogeneity existed, data was analyzed using a random effects model. In the absence of heterogeneity, a fixed effects model was used. The RR was considered significant when $P$ value was less than 0.05 (2-tailed), with values of $<1$ favoring concurrent chemoradiotherapy. The presence of publication bias was assessed with the Begg's [15] and Egger's tests [16].

\section{Results}

\section{Patients characteristics and quality assessment}

Through the electronic databases, a total of 1968 citations were searched. Initially, 932 studies were excluded for duplicate. After screening through titles and abstracts, a total of fifty-two randomized controlled trials (RCTs) investigating CCRT versus radiotherapy in patients with NPC were identified. The data on the severe late toxicity were reported in 5 studies [17-21], which were further included in the meta-analysis. Figure 1 shows the selection process in detail.

The main characteristics of the included studies are presented in Table 1. Patients in Lee's trial [18] were randomized to receive conventional-fractionation RT alone (CF group), accelerated-fractionation RT alone (AF group), conventional-fractionation RT plus concurrentadjuvant chemotherapy ( $\mathrm{CF}+\mathrm{C}$ group), or acceleratedfractionation RT plus concurrent-adjuvant chemotherapy ( $\mathrm{AF}+\mathrm{C}$ group). Only the patients in $\mathrm{CF}$ group and $\mathrm{CF}+\mathrm{C}$ group were included in our analysis. The included RCTs had a median follow-up of at least 5 years. A total of 1102 patients with histologically proven NPC were available for the meta-analysis. There were 547 patients in CCRT group and 555 in RT group. Late toxicity in all the included trials was graded according to Late Radiation Morbidity Scoring Criteria of the Radiation Therapy Oncology Group [22]. Severe late toxicity was defined as grade 3, 4 and 5 taken together.

All the included trials reported withdrawals and dropouts. Four trials had mentioned the concealment of allocation clearly in the randomization process and had Jadad scores of 3. One trial did not mention the method of generating sequence of randomization and had Jadad scores of 2 .

\section{Incidence and RR of overall severe late toxicities}

For total types of severe late toxicities, there were 150 events among 547 NPC patients that received CCRT, conferring a summary incidence of $30.7 \%$ (95\% CI, 18 47.2\%; heterogeneity test: $\mathrm{P}<0.001 ; \mathrm{I}^{2}=47.7 \%$ ). There were 118 events among 555 patients receiving RT alone, with an incidence of $21.7 \%$ (95\% CI, 13.3-33.4\%; heterogeneity test: $\left.\mathrm{P}<0.001 ; \mathrm{I}^{2}=46.3 \%\right)$. The summary RR of overall severe late toxicities for CCRT versus RT was 1.349 (95\% CI, 1.108-1.643; $\mathrm{P}=0.005$ ) (Figure 2), 


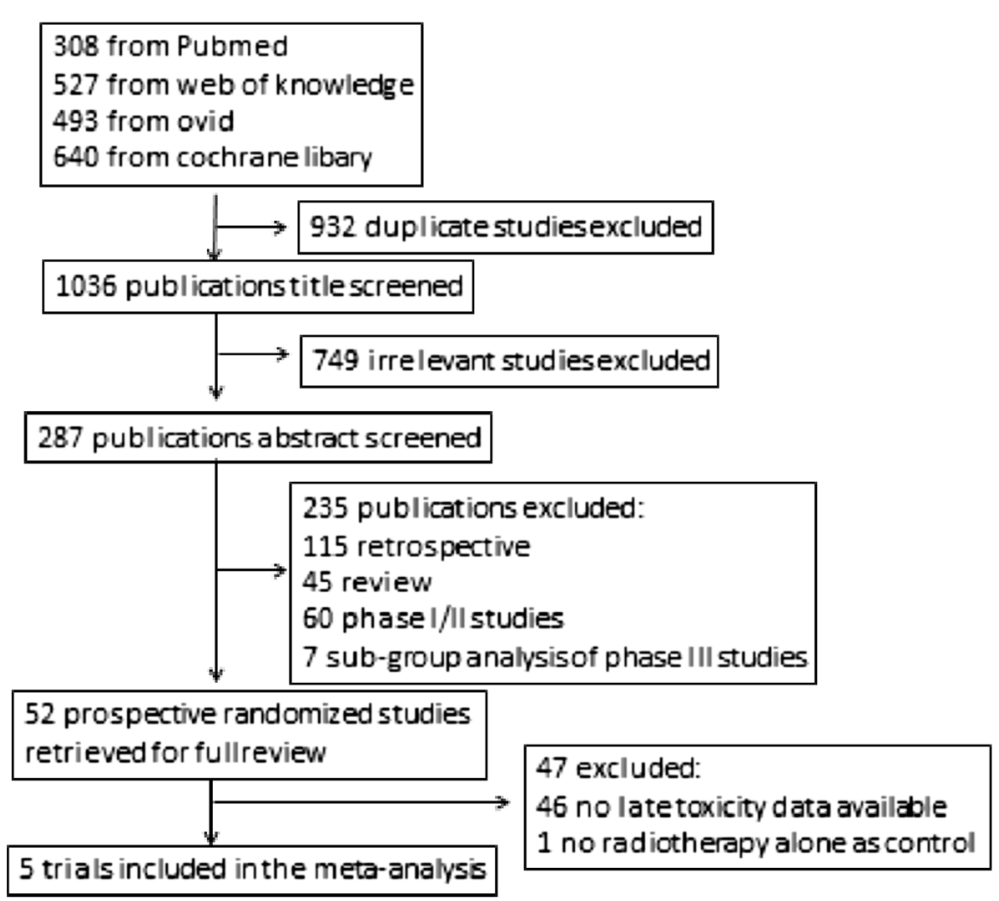

Figure 1 CONSORT diagram: flow chart of trail selection process for the meta-analysis.

suggesting a significant increase of severe late toxicities in NPC patients receiving CCRT. No significant heterogeneity was observed $\left(\mathrm{Q}=1.592 ; \mathrm{P}=0.81 ; \mathrm{I}^{2}=0.0 \%\right)$.

\section{Incidence and RR of specific late toxicity}

The pooled analysis of the incidence and RR of specific late toxicities recorded by at least 2 trials is shown in
Table 2. The main severe late toxicities included: ear deafness/otitis, cranial neuropathy, neck soft tissue damage, endocrine dysfunction. CCRT significantly increased the risk of ear deafness/otitis compared with RT $(\mathrm{RR}=1.567 ; 95 \% \mathrm{CI}, 1.192-2.052)$, but no significant difference was observed in the risk of other kinds of severe late toxicities. The included trials were stratified

Table 1 Characteristics of RCTs Included in the meta-analysis

\begin{tabular}{|c|c|c|c|c|c|c|c|c|}
\hline Study & $\begin{array}{l}\text { No. of } \\
\text { patients }\end{array}$ & $\begin{array}{l}\text { Inclusion } \\
\text { period }\end{array}$ & Stage & $\begin{array}{l}\text { Median } \\
\text { follow-up } \\
\text { (Ms) }\end{array}$ & Group & Radiotherapy & CCRT & $A C$ \\
\hline $\begin{array}{l}\text { Lee et al. } \\
\text { (2011) [19] }\end{array}$ & 93 & 1999-2004 & $\begin{array}{l}\text { AJCC stage } \\
\text { T3-4 N0-1 M0 }\end{array}$ & 75 & $\begin{array}{l}\text { CCRT + AC } \\
\text { RT }\end{array}$ & $\begin{array}{l}\geq 66 \text { Gy } \\
(2 \mathrm{~Gy} / \mathrm{Fx} / \mathrm{d}, 5 \mathrm{Fx} / \mathrm{wk})\end{array}$ & $\begin{array}{l}\text { Cisplatin } 100 \text { mg/m2 } \\
\text { intravenously every } \\
3 \text { weeks }\end{array}$ & $\begin{array}{l}\text { Cisplatin } 80 / \mathrm{m} 2+ \\
\text { fluorouracil } 1000 \mathrm{mg} / \mathrm{m} 2 / \\
\text { day by } 96-\mathrm{h} \text { infusion every } \\
4 \text { weeks for } 3 \text { cycles. }\end{array}$ \\
\hline $\begin{array}{l}\text { Lee et al. } \\
(2010) \text { [21] }\end{array}$ & 348 & 1999-2004 & $\begin{array}{l}\text { AJCC stage III } \\
\text { and IV, any T, } \\
\text { N2 or N3, M0 }\end{array}$ & 71 & $\begin{array}{l}\text { CCRT + AC } \\
\text { RT }\end{array}$ & $\begin{array}{l}\geq 66 \text { Gy } \\
(2 \mathrm{~Gy} / \mathrm{Fx} / \mathrm{d}, 5 \mathrm{Fx} / w \mathrm{w})\end{array}$ & $\begin{array}{l}\text { Cisplatin } 100 \mathrm{mg} / \mathrm{m} 2 \\
\text { every } 3 \mathrm{wks} \text { on days } \\
1,22,43\end{array}$ & $\begin{array}{l}\text { Cisplatin } 80 \mathrm{mg} / \mathrm{m} 2+ \\
\text { fluorouracil } 1000 \mathrm{mg} / \mathrm{m} 2 / \mathrm{d} \\
\text { every } 4 \text { wks on days 71,99 } \\
\text { and } 127\end{array}$ \\
\hline $\begin{array}{l}\text { Chen } \\
\text { et al. } \\
\text { (2013) [18] }\end{array}$ & 316 & 2002-2005 & $\begin{array}{l}\text { AJCC stage III } \\
\text { and IVb }\end{array}$ & 70 & $\begin{array}{l}\text { CCRT + AC } \\
\text { RT }\end{array}$ & $\begin{array}{l}\geq 66 \mathrm{~Gy} \\
(2 \mathrm{~Gy} / \mathrm{Fx} / \mathrm{d}, 5 \mathrm{Fx} / \mathrm{wk})\end{array}$ & $\begin{array}{l}\text { Cisplatin } 40 \mathrm{mg} / \mathrm{m} 2 \text { on } \\
\text { day } 1 \text { weekly }\end{array}$ & $\begin{array}{l}\text { Cisplatin } 80 \mathrm{mg} / \mathrm{m} 2 \text { on } \\
\text { day } 1 \text { and fluorouracil } \\
800 \mathrm{mg} / \mathrm{m} 2 \text { daily as an } \\
\text { intravenous, 120-hour } \\
\text { infusion) on days } 1 \\
\text { through } 5\end{array}$ \\
\hline $\begin{array}{l}\text { Chen } \\
\text { et al. } \\
\text { (2011) [20] }\end{array}$ & 230 & 2003-2007 & $\begin{array}{l}\text { Chinese } 1992 \\
\text { staging system } \\
\text { stage II }\end{array}$ & 60 & $\begin{array}{l}\text { CCRT } \\
\text { RT }\end{array}$ & $\begin{array}{l}68-70 \text { Gy } \\
(2 \mathrm{~Gy} / \mathrm{Fx} / \mathrm{d}, 5 \mathrm{Fx} / \mathrm{wk})\end{array}$ & $\begin{array}{l}\text { Cisplatin } 30 \text { mg/m2 } \\
\text { weekly }\end{array}$ & none \\
\hline $\begin{array}{l}\text { Wu et al. } \\
\text { (2013) [17] }\end{array}$ & 115 & 2001-2003 & $\begin{array}{l}\text { AJCC stage III } \\
\text { and IVb }\end{array}$ & 114 & $\begin{array}{l}\text { CCRT } \\
\text { RT }\end{array}$ & $\begin{array}{l}\text { 70-74Gy } \\
\text { (2Gy/Fx/d, 5Fx/wk) }\end{array}$ & $\begin{array}{l}\text { Oxaliplatin70 mg/m2 } \\
\text { on days } 1 \text { every week }\end{array}$ & none \\
\hline
\end{tabular}




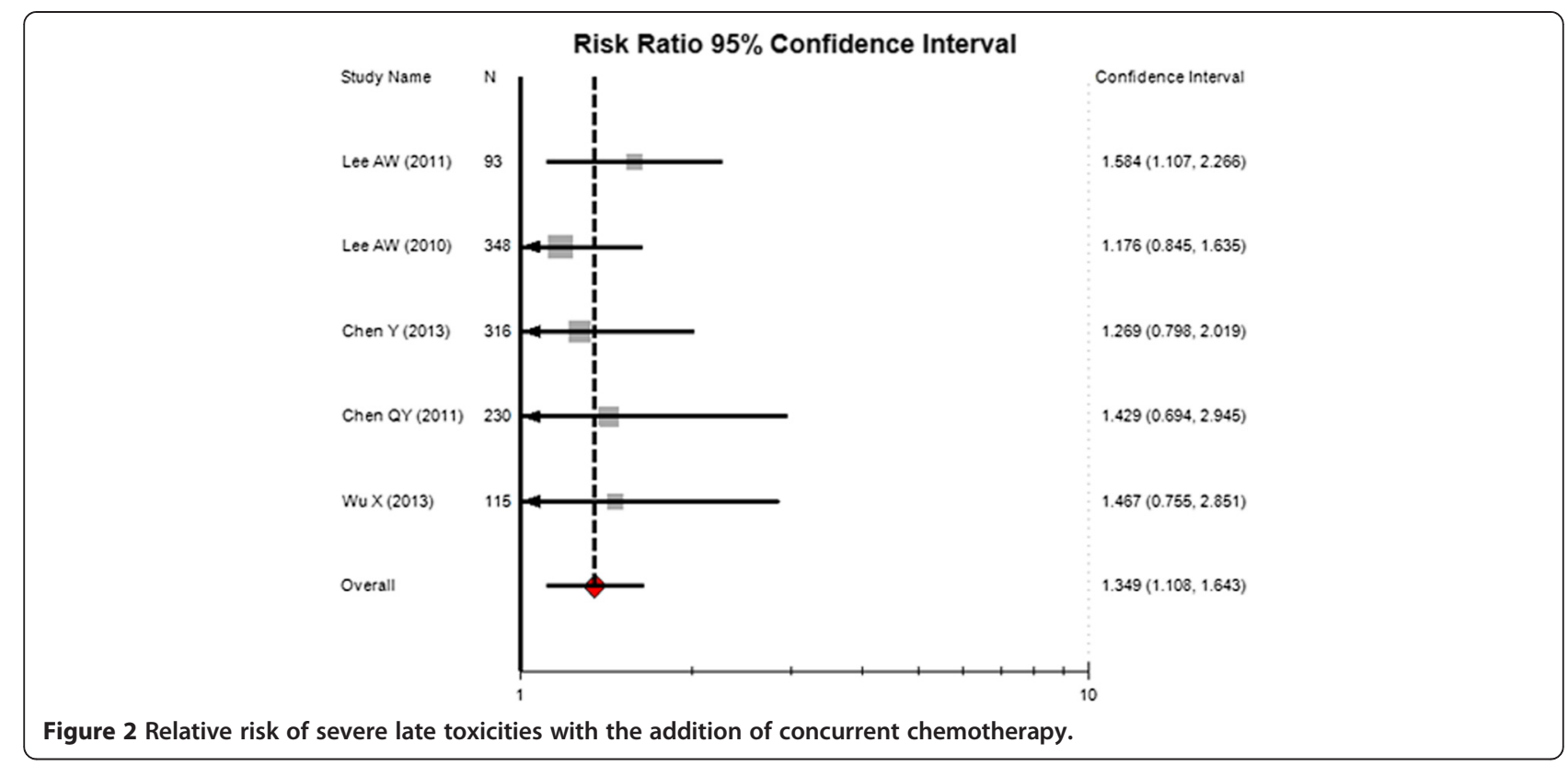

for concurrent chemotherapy regimen. Patients receiving concurrent chemotherapy regimens with 3 -week high-dose cisplatin $(\mathrm{HC})$ have a higher risk of ear deafness ( $R R=1.672$; 95\% CI, 1.174-2.382; $\mathrm{P}=0.026)$. However, there was no significant increase in the $R R$ of severe ototoxicity with the addition of non-3-week high-dose cisplatin (nonHC) regimens $(\mathrm{RR}=1.433$; 95\% CI, 0.946-2.171; $\mathrm{P}=0.095$ ) (Table 3).

\section{Publication bias}

There was no evidence of publication bias for RR of overall severe late toxicities by either the Begg's or the
Egger's test (Begg's $\mathrm{P}=0.806$ and Egger's $\mathrm{P}=0.687$ ). No publication bias for RR of severe deafness/otitis was observed (Begg's $\mathrm{P}=0.806$ and Egger's $\mathrm{P}=0.696$ ).

\section{Discussion}

As far as we know, this is the first meta-analysis evaluating the incidence and risk of severe late toxicities associated with CCRT. In current study, we reviewed the existing data on the severe late toxicities from RCTs in NPC patients by a meta-analytical method. In this analysis, all the included RCTs have a median follow-up of at least 5 years, which was considered to be a useful

Table 2 Incidence and relative risk (RR) of specific late toxicity

\begin{tabular}{|c|c|c|c|c|c|c|}
\hline \multirow[t]{2}{*}{ Late toxicities } & \multirow[t]{2}{*}{ No. of studies } & \multicolumn{2}{|c|}{$\begin{array}{l}\text { No. of severe late toxicity/total } \\
\text { no. of patients }\end{array}$} & \multicolumn{2}{|c|}{$\begin{array}{l}\text { Incidence of severe late toxicity, } \\
\%(95 \% \mathrm{Cl})\end{array}$} & \multirow[t]{2}{*}{$\mathbf{R R}$} \\
\hline & & Chemoradiotherapy & Radiotherapy & Chemoradiotherapy & Radiotherapy & \\
\hline Overall late toxicities & 5 & $150 / 547$ & $118 / 555$ & $30.7(18-47.2)$ & 21.7(13.3-33.4) & 1.349(1.108-1.619) \\
\hline Ear (deafness/otitis) & 5 & $107 / 547$ & $72 / 555$ & $20.7(12-33.2)$ & 13.3(9.3-18.7) & 1.567(1.197-2.052) \\
\hline Cranial neuropathy & 4 & $19 / 431$ & $21 / 441$ & $5.5(3.6-8.5)$ & $5.2(3.4-7.9)$ & $0.923(0.351-2.427)$ \\
\hline Soft tissue damage at neck & 4 & $17 / 431$ & $17 / 441$ & $4.6(2.9-7.2)$ & $4.2(2.6-6.6)$ & $1.007(0.522-1.94)$ \\
\hline Peripheral neuropathy & 3 & $6 / 372$ & $2 / 385$ & $1.8(0.9-3.9)$ & $1.6(0.5-5)$ & $2.295(0.41-12.845)$ \\
\hline Temporal lobe necrosis & 2 & $5 / 200$ & $11 / 209$ & $2.9(1.3-6.5)$ & $5.8(3.2-10.2)$ & $0.47(0.079-2.809)$ \\
\hline Brachial plexopathy & 2 & $0 / 214$ & $1 / 227$ & $0.6(0.1-4.1)$ & $0.7(0.1-3.3)$ & $0.567(0.048-6.707)$ \\
\hline Endocrine dysfunction & 2 & $15 / 214$ & $12 / 227$ & $7.1(4.3-11.4)$ & $5.3(3.1-9.2)$ & $1.312(0.629-2.74)$ \\
\hline Visual toxicity & 2 & $3 / 200$ & $3 / 209$ & $2.4(0.8-7.2)$ & $2.1(0.7-6.4)$ & $1.14(0.236-5.507)$ \\
\hline Dysphagia & 2 & $2 / 214$ & $0 / 227$ & $1.2(0.3-3.9)$ & $0.5(0.1-3.6)$ & $2.973(0.272-32.51)$ \\
\hline Bone necrosis & 2 & $2 / 330$ & $1 / 334$ & $0.6(0.2-2.4)$ & $0.5(0.1-2.3)$ & 1.622(0.201-13.111) \\
\hline Mucosal damage & 2 & $4 / 231$ & $1 / 232$ & $2.1(0.8-5.1)$ & $0.7(0.1-3.2)$ & $2.891(0.431-19.409)$ \\
\hline Radiation-induced malignancy & 2 & $0 / 330$ & $1 / 333$ & $0.3(0-2.1)$ & $0.5(0.1-2.3)$ & $0.524(0.044-6.226)$ \\
\hline
\end{tabular}


Table 3 Relative risk of severe ototoxicity by concurrent chemotherapy regimen

\begin{tabular}{|c|c|c|c|c|c|}
\hline \multirow[t]{2}{*}{ Study } & \multicolumn{2}{|c|}{ No. of events/patient size } & \multirow[t]{2}{*}{ Relative risk } & \multirow[t]{2}{*}{$95 \% \mathrm{Cl}$} & \multirow[t]{2}{*}{$P$ value } \\
\hline & CCRT & RT & & & \\
\hline \multicolumn{6}{|l|}{$\overline{\mathrm{HC}}$} \\
\hline Lee et al. (2011) [19] & $22 / 42$ & $12 / 51$ & 2.226 & 1.256 to 3.947 & \\
\hline Lee et al. (2010) [21] & $37 / 172$ & $27 / 176$ & 1.402 & 0.895 to 2.198 & \\
\hline Overall & & & 1.672 & 1.174 to 2.382 & 0.026 \\
\hline \multicolumn{6}{|l|}{ Non-HC } \\
\hline Chen et al. (2013) [18] & $28 / 158$ & $18 / 158$ & 1.556 & 0.898 to 2.695 & \\
\hline Chen et al. (2011) [20] & $12 / 116$ & $8 / 114$ & 1.474 & 0.626 to 3.471 & \\
\hline Wu et al. (2013) [17] & $8 / 59$ & $11 / 56$ & 1.085 & 0.421 to 2.794 & \\
\hline Ovarall & & & 1.433 & 0.946 to 2.171 & 0.095 \\
\hline
\end{tabular}

CCRT: concurrent chemoradiotherapy; RT: radiotherapy; HC: 3-week high-dose cisplatin; non-HC: non-3-week high-dose cisplatin.

starting point for reporting late toxicity. It's revealed in our analysis that NPC patients treated with CCRT had a significantly increased risk of severe late toxicities compared with those given with RT alone. The incidence of severe late toxicities for patients receiving CCRT was $30.7 \%$ compared with $21.7 \%$ for those treated with RT. Whether the addition of concurrent chemotherapy increases the incidence and severity of late toxicities in NPC patients was controversial in the literature. These findings could be explained by that the published data on late toxicities induced by CCRT were usually from studies that are small sample sizes, retrospective, involved treatment heterogeneity, or with inadequate follow-up [10-12].

Ototoxicity is the most common severe late toxicity, accounting for almost two thirds of total severe late toxicities in this analysis. CCRT incurred a significant 1.567fold increase in the risk of severe ototoxicity, which is not surprising because both cisplatin and RT are known to cause hearing loss. However, with weekly intermediate dose of cisplatin or oxaliplatin (nonHC), the patients didn't experience a statistically significant increased risk. No additive effect for weekly intermediate dose of cisplatin on ototoxicity may be explained by that the dose level of cisplatin given per infusion was too low to induce ototoxicity by itself [23]. Although $\mathrm{HC}$ regimens had resulted in favorable outcome for locally advanced NPC, it was associated with increased acute and poor compliance. In the Intergroup 0099 study, only $63 \%$ of patients who were scheduled to receive three courses of concurrent $100 \mathrm{mg} / \mathrm{m} 2$ cisplatin actually did so. This issue may be overcome by the change of combination schedule of cisplatin or the use of other anticancer agents. It's reported [24] that a weekly intermediate dose of cisplatin could decrease interruptions in radiation treatment and reduce acute toxic effects without compromising local control. Weekly oxaliplatin combined concomitantly with RT was also found to achieve a significant improvement in therapeutic outcome with minimal and acceptable additional toxic effects [14]. Thus, nonHC regimens may be an alternative of treatment for NPC in order to reduce the rate of ototoxicity.

Except for ototoxicity, the risk of other severe late toxicities didn't increase with the addition of concurrent chemotherapy in this analysis, for which we suggest several possible explanations: the small number of events recorded; underreporting of rare adverse events; the fact that clinical trials are usually not designed specifically to address toxic events; and the small number of randomized controlled trials included.

There are some limitations in this meta-analysis. First, the follow-up duration of the included trials was inadequate for assessing all types of late toxicities. Five year follow-up is long enough to assess the development of ototoxicity but may be inadequate for neurological complications, the median latency of which exceeds 5 years. Kong et al. [25] reported that the occurrence of cranial neurophathy increased with prolonged follow-up, reaching $44.5 \%$ at 20 years. But the included data in this analysis is the most updated data we can get from RCTs. This limitation can only be overcome by the further update of the RCTs. Second, the number RTCs included in this analysis is small. We have searched the Web of knowledge, Embase, Cochrane controlled trials register and Pubmed updated to August 2014, but only five RCTs fulfilled the inclusion criteria. The data on severe late toxicities are unavailable in most of RCTs. Thus the updates of long term results from RCTs are encouraged to provide a better understanding of the potential adverse impacts of CCRT on NPC patients. Third, this meta-analysis is based on published data and tends to overestimate treatment effects compared with individual patient data analyses.

\section{Conclusion}

This study demonstrates that the use of CCRT seems to increase the risk of severe late toxicities in patients with 
NPC, especially when using $\mathrm{HC}$ regimen for the occurrence of severe ototoxicity. But one should be cautious when interpreting these results due to the limitations of our study. Additionally, as concurrent chemotherapy with cisplatin gains greater clinical use, clinicians should be aware of the risks of severe late toxicities with the addition of high dose cisplatin and adequately explain these risks to gain truly informed consent of our patients.

\section{Abbreviations}

NPC: Nasopharyngeal carcinoma; CCRT: Concurrent chemoradiotherapy; QoL: Quality of life; RR: Relative risk; RCTs: Randomized controlled trials; HC: 3-week high-dose cisplatin; nonHC: Non-3-week high-dose cisplatin.

\section{Competing interest}

The authors declare that they have no competing interests

\section{Authors' contributions}

$H Y, C D$ and $C H$ designed the study. CD, FK and RZ aided in data collection. $\mathrm{CD}$ performed the statistical analysis and draft the manuscript. All authors read and approved the final manuscript.

Received: 7 December 2014 Accepted: 9 March 2015

Published online: 26 March 2015

\section{References}

1. Lee AW, Lin JC, Ng WT. Current management of nasopharyngeal cancer. Semin Radiat Oncol. 2012;22(3):233-44.

2. Ou J, Pan F, Geng P, Wei X, Xie G, Deng J, et al. Silencing fibronectin extra domain $A$ enhances radiosensitivity in nasopharyngeal carcinomas involving an FAK/Akt/JNK pathway. Int J Radiat Oncol Biol Phys. 2012;82(4):e685-91.

3. Al-Sarraf M, LeBlanc M, Giri PG, Fu KK, Cooper J, Vuong T, et al. Chemoradiotherapy versus radiotherapy in patients with advanced nasopharyngeal cancer: phase III randomized Intergroup study 0099. J Clin Oncol. 1998;16(4):1310-7

4. Chan AT, Teo PM, Ngan RK, Leung TW, Lau WH, Zee B, et al. Concurrent chemotherapy-radiotherapy compared with radiotherapy alone in locoregionally advanced nasopharyngeal carcinoma: progression-free survival analysis of a phase III randomized trial. J Clin Oncol. 2002;20(8):2038-44.

5. Lin JC, Jan JS, Hsu CY, Liang WM, Jiang RS, Wang WY. Phase III study of concurrent chemoradiotherapy versus radiotherapy alone for advanced nasopharyngeal carcinoma: positive effect on overall and progression-free survival. J Clin Oncol. 2003;21(4):631-7.

6. Kwong DL, Sham JS, Au GK, Chua DT, Kwong PW, Cheng AC, et al. Concurrent and adjuvant chemotherapy for nasopharyngeal carcinoma: a factorial study. J Clin Oncol. 2004;22(13):2643-53.

7. Wee J, Tan EH, Tai BC, Wong HB, Leong SS, Tan T, et al. Randomized trial of radiotherapy versus concurrent chemoradiotherapy followed by adjuvant chemotherapy in patients with American Joint Committee on Cancer/ International Union against cancer stage III and IV nasopharyngeal cancer of the endemic variety. J Clin Oncol. 2005;23(27):6730-8.

8. Lee AW, Lau WH, Tung SY, Chua DT, Chappell R, Xu L, et al. Preliminary results of a randomized study on therapeutic gain by concurrent chemotherapy for regionally-advanced nasopharyngeal carcinoma: NPC-9901 Trial by the Hong Kong Nasopharyngeal Cancer Study Group. J Clin Oncol. 2005;23(28):6966-75.

9. Baujat B, Audry H, Bourhis J, Chan AT, Onat H, Chua DT, et al.

Chemotherapy in locally advanced nasopharyngeal carcinoma: an individual patient data meta-analysis of eight randomized trials and 1753 patients. Int J Radiat Oncol Biol Phys. 2006;64(1):47-56.

10. Tsai WL, Huang TL, Liao KC, Chuang HC, Lin YT, Lee TF, et al. Impact of late toxicities on quality of life for survivors of nasopharyngeal carcinoma. BMC Cancer. 2014;14:856.

11. Tuan JK, Ha TC, Ong WS, Siow TR, Tham IW, Yap SP, et al. Late toxicities after conventional radiation therapy alone for nasopharyngeal carcinoma. Radiother Oncol. 2012;104(3):305-11.

12. Lee AW, Ng WT, Hung WM, Choi CW, Tung R, Ling YH, et al. Major late toxicities after conformal radiotherapy for nasopharyngeal carcinoma-patient- and treatment-related risk factors. Int J Radiat Oncol Biol Phys. 2009;73(4):1121-8.
13. Petsuksiri J, Sermsree A, Thephamongkhol K, Keskool P, Thongyai K, Chansilpa $Y$, et al. Sensorineural hearing loss after concurrent chemoradiotherapy in nasopharyngeal cancer patients. Radiat Oncol. 2011;6:19.

14. Moher D, Pham B, Jones A, Cook DJ, Jadad AR, Moher M, et al. Does quality of reports of randomised trials affect estimates of intervention efficacy reported in meta-analyses? Lancet. 1998;352(9128):609-13.

15. Begg CB, Mazumdar M. Operating characteristics of a rank correlation test for publication bias. Biometrics. 1994;50(4):1088-101.

16. Egger M, Davey Smith G, Schneider M, Minder C. Bias in meta-analysis detected by a simple, graphical test. BMJ. 1997;315(7109):629-34.

17. Wu X, Huang PY, Peng PJ, Lu LX, Han F, Wu SX, et al. Long-term follow-up of a phase III study comparing radiotherapy with or without weekly oxaliplatin for locoregionally advanced nasopharyngeal carcinoma. Ann Oncol. 2013;24(8):2131-6.

18. Chen $Y$, Sun $Y$, Liang SB, Zong JF, Li WF, Chen $M$, et al. Progress report of a randomized trial comparing long-term survival and late toxicity of concurrent chemoradiotherapy with adjuvant chemotherapy versus radiotherapy alone in patients with stage III to IVB nasopharyngeal carcinoma from endemic regions of China. Cancer. 2013;119(12):2230-8.

19. Lee AW, Tung SY, Chan AT, Chappell R, Fu YT, Lu TX, et al. A randomized trial on addition of concurrent-adjuvant chemotherapy and/or accelerated fractionation for locally-advanced nasopharyngeal carcinoma. Radiother Oncol. 2011;98(1):15-22.

20. Chen QY, Wen YF, Guo L, Liu H, Huang PY, Mo HY, et al. Concurrent chemoradiotherapy vs radiotherapy alone in stage II nasopharyngeal carcinoma: phase III randomized trial. J Natl Cancer Inst. 2011;103(23):1761-70.

21. Lee AW, Tung SY, Chua DT, Ngan RK, Chappell R, Tung R, et al. Randomized trial of radiotherapy plus concurrent-adjuvant chemotherapy vs radiotherapy alone for regionally advanced nasopharyngeal carcinoma. J Natl Cancer Inst. 2010:102(15):1188-98.

22. Cox JD, Stetz J, Pajak TF. Toxicity criteria of the Radiation Therapy Oncology Group (RTOG) and the European Organization for Research and Treatment of Cancer (EORTC). Int J Radiat Oncol Biol Phys. 1995;31(5):1341-6.

23. Chan SH, Ng WT, Kam KL, Lee MC, Choi CW, Yau TK, et al. Sensorineural hearing loss after treatment of nasopharyngeal carcinoma: a longitudinal analysis. Int J Radiat Oncol Biol Phys. 2009;73(5):1335-42.

24. Kim TH, Ko YH, Lee MA, Kim BS, Chung SR, Yoo le R, et al. Treatment outcome of cisplatin-based concurrent chemoradiotherapy in the patients with locally advanced nasopharyngeal cancer. Cancer Res Treat. 2008;40(2):62-70.

25. Kong L, Lu JJ, Liss AL, Hu C, Guo X, Wu Y, et al. Radiation-induced cranial nerve palsy: a cross-sectional study of nasopharyngeal cancer patients after definitive radiotherapy. Int J Radiat Oncol Biol Phys. 2011;79(5):1421-7.

\section{Submit your next manuscript to BioMed Central and take full advantage of:}

- Convenient online submission

- Thorough peer review

- No space constraints or color figure charges

- Immediate publication on acceptance

- Inclusion in PubMed, CAS, Scopus and Google Scholar

- Research which is freely available for redistribution 